\title{
Examining the influence of picture format on children's naming responses
}

\author{
Naroa Martínez ${ }^{\text {Corresp., }}{ }^{,}$, Helena Matute ${ }^{1}$ \\ ${ }^{1}$ Departamento de Fundamentos y Métodos de la Psicología, University of Deusto, Bilbao, Spain \\ Corresponding Author: Naroa Martínez \\ Email address: naroa.martinez@deusto.es
}

Digital photography has facilitated the use of more ecological stimuli than line drawings as experimental stimuli. However, there is lack of evidence regarding the effect of the picture format on children's naming agreement. The present work investigated whether the format of presentation of the pictures (line drawing or photograph) affects naming task performance in children. Two naming task experiments are reported using 106 concepts depicted both as a photograph and as a matched drawing delineated directly from the photograph. Thirty-eight and thirty-four Spanish-speaking children from 8 to 10 years old participated in Experiment 1 and Experiment 2 respectively. We examined name agreement measures ( $H$ index, percentage of modal name, and alternative responses) and subjective scales (familiarity and visual complexity). The results revealed a significant main effect of format in all of the variables except for familiarity, indicating better name agreement indices and higher visual complexity values for the photograph format than for the line drawing format. Additionally, line drawings were more likely to produce alternative incorrect names. The implications of these findings for psychoeducational research and practice are discussed. 
1

\section{Examining the influence of picture format on}

\section{3 children's naming responses}

4 Naroa Martínez ${ }^{1}$, Helena Matute ${ }^{1}$

5

$6{ }^{1}$ Departamento de Fundamentos y Métodos de la Psicología, University of Deusto, Bilbao, Spain

7

8 Corresponding Author:

9 Naroa Martínez ${ }^{1}$

10 Avda. Universidades 24, Bilbao, 48007 Spain

11 Email address: naroa.martinez@deusto.es 


\section{Abstract}

13 Digital photography has facilitated the use of more ecological stimuli than line drawings as 14 experimental stimuli. However, there is lack of evidence regarding the effect of the picture

15 format on children's naming agreement. The present work investigated whether the format of 16 presentation of the pictures (line drawing or photograph) affects naming task performance in 17 children. Two naming task experiments are reported using 106 concepts depicted both as a 18 photograph and as a matched drawing delineated directly from the photograph. Thirty-eight and 19 thirty-four Spanish-speaking children from 8 to 10 years old participated in Experiment 1 and 20 Experiment 2 respectively. We examined name agreement measures $(H$ index, percentage of 21 modal name, and alternative responses) and subjective scales (familiarity and visual complexity).

22 The results revealed a significant main effect of format in all of the variables except for

23 familiarity, indicating better name agreement indices and higher visual complexity values for the

24 photograph format than for the line drawing format. Additionally, line drawings were more

25 likely to produce alternative incorrect names. The implications of these findings for 26 psychoeducational research and practice are discussed. 


\section{Introduction}

Pictures play an important role in psychoeducational assessment, intervention, and

30

research. For instance, picture naming is a very frequently used task because it allows for exploring various cognitive processes such as perceptual processing, activation of semantic information, lexical selection, name retrieval, and motor planning (see Bonin, Méot, Lagarrigue, \& Roux, 2015; Humphreys \& Riddoch, 2006; Levelt, Roelofs, \& Meyer, 1999; Riddoch \& Humphreys, 2001; Roelofs \& Ferreira, in press). In children, pictures — as opposed to written stimuli — could be the only option when conducting researching with pre-reader kindergarten pupils. Pictures might also be considered more age-appropriate for primary school children, since they exclude possible effects derived from reading skills (see Perfetti, Finger, \& Hogaboom, 1978).

One important line of research, which has provided well-controlled stimuli for picture naming tasks, consists of normative studies. Norms offer information on the variables of central relevance that influence naming performance. A pioneering normative study is the one reported by Snodgrass and Vanderwart (1980). This consisted of 260 black-and-white line drawings with norms for name agreement, image agreement (or the degree of agreement between the mental image and the picture), familiarity, and visual complexity of English-speaking adults. The results showed that pictures were named more accurately when a particular word represented by a picture was frequent and familiar, when the picture had low subjective visual complexity, and when the image agreement was high. Since then, vast literature has emerged in which these line drawing sets have been extended and adapted to numerous languages for use with adults (e.g., Sanfeliu \& Fernández, 1996; in Castilian Spanish; Weekes, Shu, Hao, Liu, \& Tan, 2007; in Chinese; Alario \& Ferrand, 1999; in French; Nisi, Longoni, \& Snodgrass, 2000; in Italian; Pind, 
51 Jonsdottir, Gossurardottir, \& Jonsson, 2000; in Icelandic; Nishimoto, Miyawaki, Ueda, Une, \&

52 Takahashi, 2005; in Japanese; Van Schagen, Tamsma, Bruggemann, Jackson, \& Michon, 1983;

53 in Dutch), and in children (e.g., Wang, Chen, \& Zhu, 2014; in Chinese; Piñeiro, Manzano, \&

54 Reigosa, 1999; in Cuban Spanish; Berman, Friedman, Hamberger, \& Snodgrass, 1989;

55 Cycowicz, Friedman, Rothstein, \& Snodgrass, 1997; in English; Cannard, Bonthoux, Blaye,

56 Scheuner, Schreiber, \& Trinquart, 2006; in French; D'Amico, Devescovi, \& Bates, 2001; in

57 Italian; Pompéia, Miranda, \& Bueno, 2001; in Portuguese). Some other works have been

58 dedicated to collect norms on the colored and textured version of the Snodgrass and Vanderwart

59 set (Rossion \& Pourtois, 2004; in English; Tsaparina, Bonin, \& Méot, 2011; in Russian; Raman,

60 Raman, \& Mertan, 2014; in Turkish; Dimitropoulou, Duñabeitia, Blitsas, \& Carreiras, 2009; in

61 Greek; Bakhtiar, Nilipour, \& Weekes, 2013; in Persian). It is worth mentioning that the

62 majority of the normative studies of picture naming have been based on the same set of stimuli,

63 the Snodgrass and Vanderwart (1980) set, or its corresponding colorized version (Rossion and

64 Pourtois, 2004). Very few studies have used a different set of line drawings. These are, for

65 example, the set offered by Bonin, Peereman, Malardier, Méot, \& Chalard (2003) in French; the

66 Protocole Européen de Dénomination Orale d'Images (PEDOI, Kremin, Akhutina, Basso,

67 Davidoff, De Wilde, Kitzing et al., 2003; in Dutch, English, German, French, Italian, Russian,

68 Swedish, and Spanish); or the Multilingual Picture databank (MultiPic, Duñabeitia et al., 2018)

69 in Spanish, British English, German, Italian, French and Dutch.

70 In addition to drawing format datasets, technological development has facilitated the use

71 of digital photographs as experimental stimuli. Moreover, the standardization of photographic

72 sets for adults has progressively increased in recent years (see, for example, Adlington, Laws, \&

73 Gale, 2009; and Russo, Hagmann, Andrews, Black, Silberman, \& Shea, 2018; in English; 
74 Brodeur, Dionne-Dostie, Montreuil, \& Lepage, 2010; and Brodeur, Guérard, \& Bouras, 2014; in

75 English and French; Saryazdi, Bannon, Rodrigues, Klammer, \& Chambers, 2018; in Turkish;

76 Shao \& Stiegert, 2016; in Dutch; Moreno-Martínez \& Montoro, 2012; in Spanish; Navarrete,

77 Arcara, Mondini, \& Penolazzi, 2019; in Italian). Thus, the need for more ecological stimuli than

78 those provided by line drawings has begun to be highlighted. However, to the best of our

79 knowledge, no standardized set of photographs with norms for children has yet been published.

80 For this reason, we recently created a new bank with both photographs and matched line

81 drawings with norms for children (Martínez, Matute, \& Goikoetxea, 2019).

82 In the present research we aim to test whether significant differences exist as a function

83 of picture format (i.e., photographs vs. drawings) on picture naming tasks. Both line drawings

84 and photographs have different characteristics that should affect object recognition and naming

85 responses. Line drawings are schematic, simple, and prototypical representations of concepts

86 whilst photographs offer a realistic representation including color and surface details such as

87 texture, along with information about volume, brightness, and shade. Below we discuss studies

88 with adults that have revealed a picture format effect in picture naming. However, similar studies

89 are scarce for children, partly because that normative data of photographic sets have not yet been

90 collected. We will also present evidence of the photograph facilitation effect when compared

91 with the use of line drawings in children's object recognition, reported with the use of tasks other

92 than picture naming.

93 Some studies with adults have examined the effect of picture format in a picture naming

94 task by comparing drawings and photographs of different sets of stimuli (O’Sullivan, Lepage,

95 Bouras, Montreuil, \& Brodeur, 2012; Shao \& Stiegert, 2016) or using the same set of stimuli

96 (Salmon, Matheson, \& McMullen, 2014). In addition, a few studies matched also the shape, 
97 scale, and orientation features between drawings and photographs (Price \& Humphreys, 1989;

98 Brodie, Wallace, \& Sharrat, 1991; Saryazdi et al., 2018). Of those, the image types and the

99 naming variables examined differed between the different studies. For example, Price \&

100 Humphreys (1989) examined the effect of picture features on naming accuracy (the percentage

101 of error) and reaction time in three experiments by comparing five different picture formats

102 (correct color photograph, black-and-white photographs, correct color line-drawings, black-and-

103 white line drawings, and incongruent color line drawings). Among the main findings, they found

104 that both the correct color and the photographic details improved naming accuracy and reduced

105 naming reaction time. Brodie et al. (1991) found a progressive decrease in naming latencies from

106 line drawings to grayscale photographs to color photographs. They stated that surface details

107 presented in photographs (e.g., texture and three-dimensional cues) could facilitate recognition

108 and this information is typically missing in line drawings. Recently, Saryazdi et al. (2018)

109 explored differences between cliparts and colored photographs of 225 objects in several

110 measures (modal name and verb agreement measures, picture-name agreement, familiarity,

111 visual complexity, and image agreement). Cliparts were sophisticated colored drawings created

112 by editing photographs. They observed analogous results across cliparts and photographs.

113 However, even with these two similar types of stimuli, there were significantly higher ratings of

114 verb agreement and picture-name agreement for photographs than for cliparts, although these

115 differences were small in magnitude. The authors discussed the subtle differences observed in

116 terms of a visual iconicity effect, that is, the perceptual similarity between the picture and its

117 referent. In this regard, photographs make the referential relationship more transparent, which

118 could help to transfer information between the picture and the real object. Taking into account

119 the results of the above studies, it seems that the greater the difference between the line drawing 
120 and the photograph, the greater the effect of image format on object recognition. That is, if the

121 line drawings are not created directly from the photographs and do not incorporate color and

122 surface details, it is possible to observe higher naming accuracy scores and faster reaction times

123 in photographs than in line drawings.

124 In fact, it has been extensively studied how color and surface details affect object

125 recognition in drawings and in photographs, separately. For example, it has been shown that

126 color improves name agreement (Rossion \& Pourtois, 2004), memorization (Vernon \& Lloyd-

127 Jones, 2003), and naming speed of objects (e.g., Bonin, Méot, Laroche, Bugaiska, \& Perret,

128 2019; Rossion \& Pourtois, 2004) by comparing naming performance when using black-and-

129 white, grayscale, and colored drawings; and identification and memorization of objects by

130 comparing black-and-white photographs and colored photographs (e.g., Lloyd-Jones \&

131 Nakabayashi, 2009; Uttl, Graf, \& Santacruz, 2006). In a meta-analysis, Bramão, Reis, Petersson,

132 and Faísca (2011) examined the effect of color on object recognition, mainly using naming tasks,

133 in 35 experiments involving 1,535 adult participants. They found a moderate effect of color on

134 the recognition of the object in line drawings, photographs, and photographs without superficial

135 details. A recent study conducted by Bonin et al. (2019) examined the effect of color and the role

136 of the surface details in naming performance. This study compared written naming latencies of

137 the same objects in black-and-white (Snodgrass \& Vanderwart, 1980), grayscale and colored

138 drawings (Rossion \& Pourtois, 2004). They found that colored drawings yield shorter written

139 naming latencies than grayscale drawings, and these, in turn, shorter latencies than black-and-

140 white drawings. The inclusion of grayscale texture and shading without color did not reliably

141 improve naming performance as indexed by name agreement scores, a result that agrees with

142 Rossion \& Pourtois' (2004) findings.

Peer) reviewing PDF | (2019:02:35015:1:1:NEW 17 Jul 2019) 
144 manual exploration in 9-month-old infants (Pierroutsakos \& DeLoache, 2003), imitative

145 performance for a novel action on the basis of a picture-book interaction in 18-month-old infants

146 (Simcock \& DeLoache, 2006) and in success in matching real objects with pictures in 3-year-old

147 children (Callaghan, 2000). With respect to visual iconicity, studies carried out with children

148 from 1 to 3 -years of age showed that they were better able to relate the image to the object when

149 images were more iconic in comparison with images that were less realistic (Ganea, Pickard, \&

150 DeLoache, 2008).

151 To summarize, line drawings and photographs both have different characteristics that

152 affect naming performance in adults and imitative and matching performance in children.

153 However, to the best of our knowledge, there is a lack of studies carried out with children to

154 examine the effect of the picture format on picture naming tasks. The aim of the present study

155 was to compare several variables (the name agreement scored according to the $H$ index and the 156 percentage of the modal name, the alternative names and unknown responses classified into 157 different categories, familiarity, and visual complexity) in picture naming tasks with children 158 using both line drawing and photographic stimuli. Thus, we explored the possible differences 159 between the simplest and most schematic form of a set of pictures (line drawings) with a more 160 ecological, visually iconic, and complete two-dimensional format of such pictures (photographs).

\section{Experiment 1}

\section{Materials \& Methods}

\section{Participants.}

A total of 38 native Spanish-speaking children participated in the study: 17 were from the 
166 grade $(43 \%$ girls, $M$ age $=9$ years 4 months, $S D=5.10$ months $)$. None of the participants had

167 received a diagnosis of neurological damage or problems with speaking or language. Two

168 additional children were excluded from the sample for not completing the task. All of the

169 children attended a public school in Madrid that serves families with a middle-low socio-

170 economic level. The written informed consents of the adults responsible for the children who

171 participated in the study were collected (see supplementary materials), and all children agreed

172 verbally to take part in the study.

173 The sample was selected according to the following considerations. Primary school

174 children are a sector of the population that requires well-controlled visual stimuli and one with

175 which pictures are widely used in educational materials and assessment instruments (e.g., test

176 WISC-IV; Wechsler, 2003). In addition, data collection through a written naming task facilitates

177 conducting research with large groups of participants efficiently (e.g., Berman et al., 1989; Bonin

178 et al., 2019). It also reduces significantly the time and cost of the experiments with children, as

179 long as children have the basic spelling skills that allow them to write words fluently. In this

180 respect, 3er and 4th graders constitute a good sample because by the end of the second grade

181 Spanish-speaking children achieve a basic level of spelling proficiency (Defior Jiménez-

182 Fernández, \& Serrano, 2009). In fact, a pilot study of this experiment was carried out with

183 children 5-7 years-old. We decided to conduct the pilot study individually due to the

184 considerable differences in writing speed among the participants. In addition, we observed that

185 fatigue might be a problem, so we decided to split the tasks in three phases, which further

186 lengthened the time of data collection. Moreover, a recent study by Schmetz, Magis, Detraux,

187 Barisnikov, and Rousselle (2018) assessed how basic visual processes progress in 215 children

188 from 4 to 14 years old and in 20 adults. The results showed that processing of surfaces reached 
189 190 191 192 193 194 195 196 197 198 199 200 201 202 203 204 205 206 207 208 209 210

maturity by the age of 9-10 years, processing of length and position by the age of 13-14 years, and orientation processing continues to improve beyond the age of 14 years.

\section{Ethics statement.}

The ethics committee of the University of Deusto approved the procedure of the present study (Ref: ETK-14/17-18).

\section{Materials.}

We used the PicPsy bank (Martínez et al., 2019). This bank consists of 106 concepts.

Each of them is depicted both as a photograph and as a matched drawing delineated directly from the photograph (see Figure 1 for a sample of the stimuli). We selected half of the stimuli from the bank for Experiment 1 and the other half for Experiment 2. We decided to divide the bank in two different sets in order to prevent fatigue and loss of attention in children (D'Amico et al., 2001) because our presenting each concept in two different picture formats doubled the number of trials. Thus, 53 concepts, each depicted as both a photograph and a drawing (106 pictures in total) were used in this experiment.

-Figure 1 about here-

In PicPsy (Martínez et al., 2019), the concepts represented by the pictures were selected taking into account different psycholinguistic variables and subjective ratings (see Table 1). The psycholinguistic variables were: lexical frequency diversity according to Spanish dictionary of word frequency in children's writing (Martínez \& García, 2004), and different length. The subjective ratings were: high familiarity indexes, high imagination indexes, high concreteness indexes according to a scale of 1 to 7 of ES-PAL, and the subjective age of acquisition under 8 years according to Alonso, Fernández, \& Díez (2015). We found no significant differences between the list of concepts used in Experiment 1 and those used in Experiment 2 in terms of the 
212 mentioned variables. Most of the concepts used in this experiment ( $70 \%$ approximately)

213 overlapped with those of Goikoetxea (2000) for $3^{\text {rd }}$ and $4^{\text {th }}$ graders, and corresponded to 25

214 different semantic categories, namely: animals, atmospheric phenomena, birds, buildings,

215 clothing, feelings, flowers, fruits, furniture, geographical accidents and natural land formations,

216 insects, kitchen utensils, light sources, mammals, parts of a house, parts of the human body,

217 reading material, tools, trees, types of boats, types of fabrics, types of food, types of professions, 218 types of relatives, units of weight.

219

220

221

222

223

224 225

226

227

228

229

230

231

232

233

234
-Table 1 about here-

All photographs in PicPsy (Martínez et al., 2019) were downloaded from free databases

photographs, the procedure followed was similar to that employed by Brodeur et al. $(2010 ; 2014)$

using Power Point and CorelDraw (Corel Corp., Ottawa, Canada). The four editing steps were:

1) cut the object from the scene, 2) blur the words, 3) resize the image to fit within a frame of 500x500 pixels, and 4) in some images, arrows or other images were added to improve the representation of the concept. For the arrangement of the photographs, we adopted the following criteria based on those described previously in the work of Snodgrass and Vanderwart (1980): a) in the case of animals or parts of the body, approximately the same number of images were shown oriented to the right and to the left; b) in the case of objects whose orientation upwards and downwards may vary (e.g., fork), the functional part was placed downwards with approximately the same number of photographs oriented to the right and to the left; and c) fine and elongated objects were oriented with a $45^{\circ}$ inclination.

The drawings in PicPsy (Martínez et al., 2019) were prepared by one of the authors who delineated photographs by hand. After that, they were digitized to follow the same editing 
235 procedure as the photographs. Once edited, each line drawing was vectorized to achieve

236 adequate resolution. Vectorization transforms a picture to vectors instead of pixels, which allows

237 for the enlargement or reduction of the image to any size without modifying its high quality, due

238 to its defined contours. The line drawings preserved the same scale, shape, and orientation as the 239 corresponding photographs.

240

241

242 243

244

245

246

247

248

249

250

251

252

253 254 follows:

255

256

257

\section{Procedure.}

Each participant completed the picture-naming task in both formats (line drawing and photograph). The order of format presentation was counterbalanced. In order to control the potential effects of order and sequence in the repeated-measures design, children were randomly assigned to one of two different groups. One group was presented the set in line drawings first, and the other group was presented with the photographs first. No significant differences were found in the age of the counterbalancing groups, $t(36)=-0.64, p=.523, d=-0.21$.

All participants were evaluated in groups by one examiner trained in the administration of the task in a quiet room of the school at the beginning of the 2017-2018 year. Stimuli were presented, one by one, at the center of a computer screen. Each picture was preceded by a fixation point (+) for $500 \mathrm{msec}$, and remained on the screen for around 5,000 msec or until the participants responded. The sequence of presentation of the stimuli was assigned randomly but the sequence remained the same for each format order. The variables for analysis as well as their corresponding instructions, which were presented written and orally to the participants, were as

Picture naming. Participants were asked to give a single name for each item by writing the first word that came to mind in order to name each picture. In cases where the name was unknown to them, they were instructed to write the initials DKN for "Don't Know the Name" (in 
258 Spanish NSP for No Sé la Palabra), DKO was used for "Don't Know the Object" (in Spanish

259 NSO for No Sé el Objeto), and TOT was used for "Tip-Of-the-Tongue” (in Spanish PDL for

260 Punta De la Lengua).

261 Familiarity. Participants were asked to rate the familiarity of the word represented by the 262 picture according to how often they daily interact with, hear, or think about the word on a scale

263 from 1(a few times) to 5 (many times). Participants were asked to rate the word itself rather than 264 the picture.

265 Visual complexity. Participants were asked to rate the visual complexity of the picture 266 according to the number of details they thought the picture had on a scale from 1(few details) to 2675 (many details).

268 Each participant completed three tasks (picture naming, familiarity rating, and visual 269 complexity rating) in both formats (line drawing and photograph). Each child, therefore, 270 responded to a total of 106 stimuli: 53 in line drawing and 53 in photographic format. These 271 three tasks are those that are usually included in picture naming studies with children (e.g., 272 Cannard et al., 2006; Berman et al., 1989; Cycowicz et al.,1997; D'Amico et al., 2001; Pompéia 273 et al., 2001). However, variables such as image agreement and image variability are not usually 274 included due to children's difficulty to understand these tasks, because they require children to 275 form and judge mental images (see Wang et al., 2014), in addition to handling many scales 276 simultaneously.

277 All children responded on a sheet of paper created for this purpose. The sheets included 278 numbered lines, one for each picture, where participants had to write down their answers for the 279 naming task. Following each line, two scales were presented where they were asked to indicate 280 with an $X$ the corresponding value for the rating of familiarity and visual complexity. The scales 
281 of familiarity and visual complexity were adapted from those proposed by Piñeiro et al. (1999).

282 The values of the scale were complemented with a visual scale composed of squares of different 283 sizes in order to facilitate the handling of the scales by the children. Thus, to represent the values 2841,3 and 5 of the scale three rectangles of sizes $1 \times 1 \mathrm{~cm}, 1 \times 3 \mathrm{~cm}$ and $1 \times 5 \mathrm{~cm}$ were presented, all of 285 which were gray added with a level of transparency of 50, 30, and 10\%, respectively. During the 286 session, the researchers gave prior instructions and two examples (not included in the set) were 287 completed in order to allow each participant to become familiar with the task and the scales.

288

289

290

291

292

293

294 295 296 297 298 299 300 modal name responses, the percentage of alternative names in each category, and the percentage of unknown responses in each category.

The $H$ index for each of the 53 drawings and each of the 53 photographs was analyzed.

The $H$ index is a statistic (Shannon \& Weaver, 1949), which was introduced by Snodgrass and Vanderwart (1980) as a name agreement index to reflect the dispersion of the name responses for each stimulus (drawing or photograph) and is calculated as follows:

$$
H=\sum_{i=1}^{k} P_{i} \log _{2}\left(1 / P_{i}\right)
$$
where $\mathrm{k}$ represents the number of different names given for each picture, and $\mathrm{P}_{\mathrm{i}}$ represents the

The responses of the participants were transcribed, and were corrected for spelling errors. Basic variants of the same name such as singular and plural forms (e.g., windows and window) were collapsed. For responses that included one or a second noun (e.g., frog or toad), the noun recovered second was excluded.

Different measures of name agreement were analyzed: the $H$ index, the percentage of 
303 only one name has been given for an image. The increase in the $H$ values indicates an increase in

304 the dispersion of the responses.

305 In addition, the percentage of participants who responded with the modal name was

306 calculated (name given by most subjects in the sample). Although both the $H$ index and the

307 percentage of modal name responses are measures of the naming agreement, the percentage only

308 indicates how dominant the modal name is in the sample while the $H$ index is sensitive to how

309 widely the responses are distributed for each of the names. Additionally, studies conducted with

310 children have suggested that caution should be exercised when using a percentage of modal

311 names alone as a name agreement measure (e.g., Cannard, Blaye, Scheuner, \& Bonthoux, 2005),

312 and so this is usually complemented with a qualitative analysis of the children's responses (e.g.,

313 Cycowicz et al., 1997). One of the concerns is that, unlike adults, the name given by most

314 children is not always identical to the expected name, and in some cases can even be incorrect.

315 For this reason, we complemented the measure with the percentage of participants who

316 responded with alternative responses in different categories. Alternative names were classified

317 within the categories developed by O'Sullivan et al. (2012), that is, based on whether they were

318 incorrect (e.g., physically similar such as bombilla $[$ bulb] for gota $[$ drop $]$ ), equivocal (i.e. non-

319 existing word such as rasca-uñas [scratch-nails] for lima [file]), or correct (i.e. synonyms such

320 as vehículos [vehicles] for transporte [transport]). Applying the same categorization rules

321 described by O'Sullivan et al. (2012), the classification of responses was made by two native

322 Spanish judges and by a third judge when a consensus could not be reached.

323 The responses DKN, DKO, and TOT were excluded from the analysis of $H$ index, of

324 percentage of modal name responses and of percentage of alternative responses because 
325 unknown responses were analyzed separately. The percentage of participants who provided the

326 unknown responses in each category was calculated.

\section{Results}

Table 2 provides descriptive statistics for the variables included in the analysis sorted by

329

330

331

332

333

334

-Table 2 about here-

A Shapiro-Wilk test of normality was significant for the name agreement measures $(p<$ .001 ), and non-significant for both subjective scales, familiarity ( $W=0.98, p=0.272)$, and visual complexity $(W=0.99, p=0.766)$, indicating that data might be deviated from normality in name agreement measures but not in subjective scales. Figure 2 displays a violin plot of each picture naming variable for both formats. The white dot shows the median, the box includes the interquartile range, and whiskers are extended to the most extreme data point. Each side of the shaded line represents a kernel density estimation indicating the probability density of the data at different values. We constructed the plots using the free web-based tool Interactive Dotplot (Weissgerber, Savic, Winham, Stanisavljevic, Garovic, \& Milic, 2017).

- Figure 2 about here-

In order to compare the name agreement measures between line drawing and photograph formats, we conducted mixed ANOVAs of $H$ Index and percentage of modal responses with Format (line drawing, photograph) as the within-subject factor and Order (line drawing photograph, photograph - line drawing) as between-subjects factors. The analysis of variance

(ANOVA) has proved to be robust when there is a deviation from the normality assumption ${ }^{1}$, and 
348 generally does not have strong effects on the Type I error rates or the power of the $F$-test 349 (Delacre, Leys, Mora, \& Lakens, 2018; Harwell et al., 1992; Tiku, 1971). For the $H$ index, the significant main effect of format, $F(1,104)=4.88, p=.029$, partial

351

352 353 354 355 356 357 358 359 361

362 363 364 365 366 367 368 369 370 $\operatorname{eta}^{2}=0.045$, indicated that values for line drawing were higher than those of the photograph format. With respect to the between-subjects factors, the main effect of order, $F(1,104)=0.31, p$ $=.581$, partial eta ${ }^{2}=0.003$, was not significant. Interaction between format and order, $F(1,104)=$ $1.02, p=.315$, partial eta ${ }^{2}=0.010$, was not significant. These results suggest that picture format had an effect on name agreement. In particular, photographs decreased the dispersion of names given in comparison with line drawings, thus indicating that this format improved name agreement. This finding is in line with other similar evidence reported in adults (i.e. Price \& Humphrey, 1989) and in young children employing different tasks other than naming, such as imitation or matching (Callaghan, 2000; Ganea et al., 2008; Simcock \& DeLoache, 2006). For the percentage of modal responses, the main effect of format, $F(1,104)=3.01, p=$ .086 , partial eta ${ }^{2}=0.028$, and the main effect of order, $F(1,104)=0.75, p=.390$, partial eta ${ }^{2}=$ 0.007, were not significant. However, a significant interaction between format and order was found, $F(1,104)=4.45, p=.037$, partial $\mathrm{eta}^{2}=0.041$, indicating that the format manipulations had a differential impact as a function of whether photograph or line drawing was presented first or second. To further explore the format by order interaction, we carried out a separate dependent sample $t$-test for the drawing-photograph and photograph-drawing groups to compare format differences. In the drawing-photograph order, the significant difference of format, $t(52)=$ $-2.45, p=.018, d=-0.68$, indicated that percentage of modal names for the photograph format $(M=76.68 \% ; S D=22.81)$ was higher than that of the line drawing format $(M=72.57 \% ; S D=$ 24.47). In the photograph-drawing order, the percentage difference of modal responses between 
371 formats, $t(52)=0.30, p=.764, d=0.08$, was not significant. The reported statistical interaction

372 revealed that, in modal name agreement, the format effect was likely to disappear when

373 photographs were presented before line drawings. It is noteworthy to mention that while the

374 effect of format for the $H$ index was reliable, the effect of format in the percentage of modal

375 names failed to reach statistical significance in the photograph-drawing order. As we have

376 already mentioned, the percentage of modal name responses is not sensitive to the dispersion of

377 the responses as it only indicates the dominance of the name given by most subjects (e.g.,

378 Snodgrass \& Vanderwart, 1980), and in children it should be interpreted with caution and

379 complemented with other qualitative analyses (Cycowicz et al., 1997). Indeed, in our results

380 children differed from the names intended by the experimenters (the word that the researchers

381 used for the search and selection of the picture) nine of the 53 modal names $(17 \%)$ in line

382 drawings, and eight of 53 modal names (15\%) in photographs. Of these, some of the modal

383 names were even incorrect names such as carta [letter] for sobre [envelope], and rana [frog] for 384 sapo [toad].

Given that the percentage of modal names provides limited information, particularly in samples with children (Cannard et al., 2005), we complemented the analyses by using the percentage of alternative names and the percentage of unknown responses. In order to examine differences between line drawings and photographs, a dependent sample $t$-test was conducted for each category respectively. For the alternative name responses, the dependent $t$-test revealed a significant difference in the mean percentage of incorrect names between formats, $t(105)=2.39$, $391 p=.018, d=0.47$, indicating that the average scores for line drawing were higher than those for 392 photographs. The difference in mean percentage scores between formats for equivocal names $t(105)=0.09, p=.932, d=0.18$, , and correct names $t(105)=-0.05, p=.957, d=-0.01$, were not 
394 significant. As indicated by the alternative name analysis, stimuli presented as line drawings 395 elicited similar correct and equivocal names but more incorrect names than stimuli presented in 396 photograph format. These findings suggest that the surface and color information that is missing 397 in line drawings increases visual ambiguity and evokes conceptual errors. For the unknown 398 responses, the results revealed no significant difference between formats in terms of the average 399 percentage of DKN responses, $t(105)=-1.78, p=.079, d=-0.35$, average percentage of DKO, $400 t(105)=-1.44, p=.153, d=-0.28$, and the average percentage of TOT, $t(105)=-1.76, p=.081$, $401 d=-0.34$, therefore indicating that picture format did not affect the percentage of unknown 402 responses.

404 the formats on the mean familiarity scores, $t(105)=-1.01, p=.315, d=-0.20$, was not 405 significant, but there was a significant difference between formats in terms of the mean visual 406 complexity scores, $t(105)=-2.45, p=.016, d=-0.48$, indicating that the average scores for 407 photographs were higher than the average scores for line drawings. Photographic stimuli were 408 rated as more visually complex, suggesting that children were sensitive to the greater number of 409 details (such as surface details), presented by photographs in comparison with the line drawings.

\section{Experiment 2} stimuli and a different sample, in order to ensure that the results were reliable and generalizable.

\section{Materials \& Methods}

\section{Participants.}


417 (49\% girls, $M$ age $=9$ y ears 5 months, $S D=5.10$ months $)$. None had received a diagnosis of

418 neurological damage or problems with speaking or language. Three additional children were

419 excluded from the sample for not completing the task. Details of the school, informed consent

420 collection, and ethical approval were the same as in Experiment 1.

421

422

423

424

425

426

427

428

429

430

431

432

433

434

435

436

437

438

439

\section{Materials.}

We used the 53 concepts of the PicPsy bank (Martínez et al., 2019) that were not used in Experiment 1. Each concept depicted as both a photograph and a matched drawing delineated directly from the photograph (106 pictures total) was used.

We followed the same arrangement of the stimuli and we maintained equivalent properties in terms of the psycholinguistic variables and subjective ratings as in described in Experiment 1 (see Table 3). Like in Experiment 1, most of the concepts used in this experiment (approximately 79\%) were taken from those reported by Goikoetxea $(2000)$ for $3^{\text {rd }}$ and $4^{\text {th }}$ graders, and corresponded to 25 semantic categories (40\% of them overlapped with those of Experiment 1). The 25 categories in the present experiment were: animals, atmospheric phenomena, birds, buildings, clothing, fruits, geographical accidents and natural land formations, kitchen utensils, light sources, mammals, media, parts of the human body, reading material, tools, trees, types of boats, types of building material, types of drinks, types of fabrics, types of food, types of plants, types of professions, types of relatives, types of toys, types of vehicles.

\section{-Table 3 about here-}

\section{Procedure and scoring.}

We followed the same procedure and scoring system as described in Experiment 1 and no significant differences were found in the age of the counterbalancing groups, $t(32)=-0.28, p=$ $.782, d=-0.10$ 


\section{Results}

441

442

443

444

445

446

447

448

449

450

451

452

453

454

455

456

457

458

459

460

461

462

Table 4 provides descriptive statistics for the variables included in the analysis, sorted by

format. A Shapiro-Wilk test of normality was significant for the name agreement measures $(\mathrm{p}<$ .001 ), and non-significant for the subjective scale of visual complexity ( $W=0.99, p=0.539$ ), indicating that data might be deviated from normality in name agreement measures but not in subjective scales.

-Table 4 about here-

In order to compare the name agreement measures between line drawings and photographs, we conducted mixed ANOVAs ${ }^{2}$ of $H$ Index and percentage of modal responses with Format (line drawings, photographs) as the within-subject factor and Order (line drawing photograph, photograph - line drawing) as between-subject factors. Figure 3 displays a violin plot of each picture naming variable for both formats.

-Figure 3 about here-

For the $H$ index, the significant main effect of format, $F(1,104)=13.80, p=.000$, partial eta $^{2}=.117$, indicated that values for line drawing were higher than those for the photograph format. With respect to the between-subject factors, the main effect of order, $F(1,104)=0.75, p$ $=.387$, partial eta ${ }^{2}=0.007$, was not significant, whilst the interaction between format and order, $F(1,104)=2.43, p=.122$, partial eta ${ }^{2}=0.023$, also failed to reach significance. The replication of the format effect in the $H$ index is indicative of a higher name agreement for photographs in comparison with line drawings.

For the percentage of modal responses, a significant main effect of format, $F(1,104)=$ 7.07, $p=.009$, partial eta $^{2}=0.064$, was found. With respect to the between-subjects factors, the main effect of order, $F(1,104)=1.06, p=.306$, partial eta ${ }^{2}=0.010$, was not significant. A 
463 significant interaction between format and order, $F(1,104)=4.48, p=.037$, partial eta $^{2}=0.041$, 464 was found. In the drawing-photograph order, the significant effect of format, $t(52)=-2.92, p=$. $465005, d=-0.81$, indicated that the percentage of modal name responses for the photograph format $466(M=83.22 \% ; S D=18.28)$ was higher than those for the line drawing format $(M=78.03 \% ; S D=$ 467 22.31). In the photograph- drawing order, the difference between formats in terms of percentage 468 modal responses, $t(52)=-0.47, p=.640, d=-0.13$, was not significant. Moreover, children 469 differed from the names intended by the experimenters seven of the 53 modal names (13\%) in 470 line drawings, and five of 53 modal names (9\%) in photographs. Of them, some of the modal 471 names were even an incorrect name such as papel [paper] for tela [cloth], or mujer [woman] for 472 beso [kiss]. As in Experiment 1, exploration of the significant interaction between format and 473 order revealed that the effect of format was significant only in the drawing-photograph order. 474 Previous studies have already documented greater naming accuracy for photographs compared 475 with line drawings in adults (e.g., Price \& Humphrey, 1989), suggesting that photographs 476 provide information such as color and surface details, which can facilitate naming. Therefore, a 477 possible explanation of the same interaction found in Experiment 1 and Experiment 2 could be 478 that children's naming of a line drawing could be affected by the name given previously to the same stimulus presented in photograph format, reaching similar levels of name agreement in 480 both conditions. However, children who named a photograph could be affected by the name 481 given previously to the same stimulus in line drawing format, but the photograph provided them 482 with more information to reach a significantly higher level of name agreement in photographs. 483 We complemented the analyses with the percentage of alternative names and the 484 percentage of unknown responses in each category, respectively. For the alternative name 485 responses, a dependent $t$-test revealed that the formats differed significantly in terms of the mean 
486 percentage of incorrect names, $t(105)=3.21, p=.002, d=0.63$, indicating that the average 487 scores for line drawing were higher than those for photographs. The difference between formats 488 in terms of mean percentage of equivocal names $t(105)=1.10, p=.275, d=0.21$, and 489 percentage of correct names $t(105)=-0.93, p=.355, d=-0.18$, failed to reach significance. The 490 higher percentage of incorrect names given to the line drawings in comparison with the 491 photographs replicates the effect previously obtained in Experiment 1. For the unknown responses, the results revealed that the formats did not differ significantly in terms of the average percentage of DKN responses, $t(105)=0.12, p=.905, d=$ 0.02 , and average percentage of DKO, $t(105)=-1.64, p=.104, d=-0.32$. However, a significant difference between formats in the percentage of TOT responses, $t(105)=2.78, p=.006, d=$ 0.54 , indicates that the average scores for line drawing were higher than those for photographs. In line with the findings of Experiment 1, the percentage of unknown responses was similar in both picture formats, except for the TOT category. However, unlike in Experiment 1, the percentage of unknown TOT responses was greater for line drawings than for photographs. Finally, for the subjective scale measures, dependent t-tests revealed that the difference 501 between the formats on the mean familiarity score, $t(105)=-1.42, p=.159, d=-0.28$, did not 502 reach significance, whilst the formats differed significantly in terms of the mean visual complexity score, $t(105)=-11.17, p=.000, d=-2.18$, indicating that the average scores for photographs were higher than those for line drawings. As revealed by the results of Experiment 1, photographs were rated as familiar as line drawings, but visually more complex. As we noted 506 in the introduction, line drawings are schematic and simple representations while photographs 507 offer surface details that enrich the available visual information. Previous research with adults 508 has shown that objective visual complexity is positively correlated with subjective visual 
509 complexity (Shao \& Stiegert, 2016). Our results indicate that children subjectively rated the

510 picture format to be more complex, a format that objectively incorporates more visual details.

\section{Discussion}

The purpose of the present experiments was to compare name agreement, alternative

513 names, unknown responses, and familiarity and visual complexity measures between

514 photographs and matched line drawings in Spanish-speaking children. In Experiment 1, we

515 found a general effect of picture format in children's naming accuracy and judgement of visual

516 complexity. This was replicated in Experiment 2 using a different sample and a different picture

517 set. Thus, we can summarize our main finding of the two experiments as showing higher name

518 agreement and higher visual complexity values for the photograph format than for the line

519 drawing format.

520 Photographs yield significantly more accurate naming performance than line drawings,

521 specifically, less dispersion of name responses ( $H$ index), a higher percentage of children giving

522 the modal name (only in line drawing - photograph order), and lower percentage of incorrect

523 alternative names. Several factors might be important in explaining the advantage of photographs

524 over line drawings in children's name agreement measures. For example, photographs offer

525 surface details (e.g., texture, brightness, and shade,) and color information that line drawings do

526 not, and this information facilitates recognition. Our results are in line with those from studies

527 with adults showing better performance in object recognition (higher naming accuracy, lower

528 response latency) for photographs - particularly colored photographs - than for line drawings

529 of the same objects (e.g., Brodie et al., 1991; Price \& Humphreys, 1989). It has been suggested

530 that color and surface cues activate more visual information than black-and-white pictures, and

531 trigger semantic knowledge that facilitates object recognition. Another explanation for 
532 differences in perceptual processing that influence the retrieval of the concept can be related to

533 the format of the two representations. For instance, Uttl et al. (2006) suggested that a schematic

534 representation of an object, such as a line drawing, might usually be perceived as a

535 representation of a class of object or type, rather than as a representation of an individual object

536 or token, like in the perception of photographs. Previous studies have provided support for the

537 assumption of different sensory and perceptual processes for the identification of photographs

538 and line drawings, such as strong embodiment and associations with real-world tangible objects

539 (Salmon et al., 2014; Saryazdi et al., 2018). Further, similar findings in the previous literature

540 have highlighted visual iconicity as one of the variables that particularly affects the performance

541 of both children and adults. Previous research with young children showed the impact of visual

542 iconicity on tasks involving the matching of pictures with real objects, with better performance

543 for photographs than for line drawings (Callaghan, 2000; Ganea et al., 2008). More recently, a

544 subtle iconicity effect in picture naming has also been found in adults (Saryazdi et al., 2018). Our

545 results in children using a picture-naming task showed that the more iconic representation

546 (photograph) produced, the better name agreement measures were obtained, better than the

547 measures of less iconic representation (line drawing).

$548 \quad$ Further, in the percentage of modal name responses, the format interacted significantly

549 with order. The results of Experiment 1 and Experiment 2 indicate that in the photograph-

550 drawing order, the effect of format was not significant. A possible explanation for this finding

551 could be that most of the children recovered the name of the photograph when looking at the line

552 drawing because it provided more information to facilitate naming. Previous evidence in adults

553 (e.g., Price \& Humphreys, 1989), and our results showing the format effect in the $H$ index seems

554 to support this explanation. The interaction found suggests that the modal name of a photograph 
555 is transferred quickly to the line drawing, a more schematic representation. In any case, the

556 percentage of modal names could be an incomplete measure of name agreement in children

557 (Cannard et al., 2005) and should be complemented with other quantitative and qualitative

558 measures. Indeed, our complementary analysis of alternative responses revealed that line

559 drawings elicited a statistically higher percentage of incorrect alternative names such as piedra

560 [rock] for nube [cloud], escalera [ladder] for cremallera [zipper] or abeja [bee] for mosca [fly].

561 The results from the subjective scales indicate that, broadly speaking, photographs were

562 rated as being as familiar as the matched line drawings, but visually more complex. It is

563 unsurprising that photographic stimuli were rated as more complex, given the greater details

564 presented in comparison with the line drawings. In addition, objective and subjective visual

565 complexity are positively correlated (Shao \& Stiegert, 2016). Our results did not concur with

566 those studies carried out with adults showing a similar or even higher visual complexity score for

567 line drawings than for photographs (Brodeur et al., 2010; Moreno-Martínez \& Montoro, 2012).

568 Although it is possible that adults' ratings could be different from those given by children, we

569 should interpret this finding with caution, given that these studies compare different samples and

570 sets.

571 We believe that the results of this study have several implications for the selection of

572 pictures as experimental and educational stimuli. As indicated by the differences in name

573 agreement and visual complexity measures for photographs in comparison with line drawings,

574 research on picture naming with children should pay attention to the type of stimuli employed,

575 because this variable can generate differences in perceptual processing and influence the retrieval

576 of the concept. On the one hand, line drawings have been widely used in research, assessment,

577 educational, and child literature materials. Children are thus highly familiar with such stimuli. 
578 When children are exposed to simple drawings, they gather the typical salient elements of the

579 object that help to develop figurative representations, flexibility of cognitive representation, and

580 symbolic capacity during childhood (Simcock \& DeLoache, 2006). This format also has less

581 visual complexity, which could facilitate visual processing and influence naming latencies (Shao

582 \& Stiegert, 2016) whilst also having the added benefit of substantially reducing printing costs

583 compared with colored photographs. On the other hand, colored photographs can facilitate object

584 naming and provide ecological stimuli that allow specific transference to objects. This format

585 can be an important vehicle for teaching vocabulary by virtue of a rich two-dimensional

586 representation of real objects, particularly for those stimuli that are encountered less frequently in

587 the child's environment. Current advancements in digital photography allow for color and

588 surface details to be revealed, which could reduce the ambiguity of the presented real-life

589 stimuli. Both line drawings and photographs are complementary instruments of excellent

590 educational value since the exposure to a variety of pictures plays an important role in

591 vocabulary acquisition.

\section{Conclusions}

593 The current study aims at examining the effect of picture format in name agreement

594 measures in two naming task experiments with children. The results showed that picture format

595 affected name agreement measures, types of alternative names, and visual complexity, but not

596 familiarity. These findings suggest that pictures were more likely to produce children's naming

597 agreement and be rated as more visually complex when presented as photographs than when

598 presented as line drawings. Importantly, the same results were observed using two different sets

599 of stimuli in two different samples of children, which suggests that these are robust and

600 generalizable results. Previous work found a picture format effect in adult's naming performance 
601 (e.g., Price \& Humphreys, 1989) and in young children's non-verbal tasks such as matching

602 pictures with real objects (e.g., Callaghan, 2000). The experiments presented here add evidence

603 of the role of picture characteristics in children's naming to the existing literature, using large

604 name agreement measures for each stimulus that contributes to a better understanding of the

605 effect.

606 This work presents some limitations that could serve as a basis for new lines of research.

607 In the case of our study, we matched the shape, size, orientation and contour of both formats but

608 photographs recruited significant visual information such as surface details (such as texture,

609 shade, three-dimensional cues), and particularly color information, that was not controlled.

610 Therefore, in future work we recommend exploring possible format differences by adding

611 colored drawings and black-and white-photographs to this dataset, but not grayscale line

612 drawings because these stimuli provide less stable results (Bonin et al., 2019). Despite the

613 challenges of data collection, it would be interesting to replicate this study in a sample of

614 children younger than 8 years old. Previous research has shown that children under 8 years old

615 are less efficient in picture naming than older children and adults (Cannard et al., 2005;

616 Cycowicz et al., 1997), which could show stronger differences between formats or in different

617 variables than those shown here. Moreover, the use of a repeated measure design where different

618 variables are examined in two picture formats could affect judgements on the scales used,

619 especially given the dependence between familiarity and visual complexity ratings (Forsythe,

620 Street, \& Helmy, 2017). Finally, it would be interesting to address other measures such as

621 naming speed, and examine a possible manipulability effect and other measures directly related

622 to the visual characteristics of the image such as image agreement and image variability. Even

623 though no previous work with children has examined these variables because they could be 
624 difficult tasks for primary school children to understand (Wang et al., 2014), a recent meta625 analysis in adults indicated that these two measures have a direct impact on picture processing

626 (Perret \& Bonin, 2018). Despite the mentioned limitations, we believe that future researchers and

627 practitioners could benefit from the findings reported in these experiments, particularly when 628 selecting visual stimuli to be used in naming tasks. 


\section{Acknowledgements}

630 We wish to thank Edurne Goikoetxea for critically reviewing the study proposal, and Aranzazu

631 Viñas and Mario Álvarez for their help in the classification of alternative-names responses. We

632 also would like to express our gratitude to Carlos Sainz de los Terreros School for providing

633 participants for the study and Sonia Cámara for helping in data collection. 


\section{References}

635 Adlington, R. L., Laws, K. R., \& Gale, T. M. (2009). The Hatfield Image Test (HIT): A new

636 picture test and norms for experimental and clinical use. Journal of Clinical and

637 Experimental Neuropsychology, 31(6), 731-753.

638 Alario, F. X., \& Ferrand, L. (1999). A set of 400 pictures standardized for French: Norms for

639 name agreement, image agreement, familiarity, visual complexity, image variability, and

640 age of acquisition. Behavior Research Methods, Instruments, \& Computers, 31, 531-552.

641

642

643

644

645

646

647

648

649

650

651

652

653

654

655

656

Alonso, M. A., Fernandez, A., \& Díez, E. (2015). Subjective age-of-acquisition norms for 7,039 Spanish words. Behavior Research Methods, 47(1), 268-274.

Bakhtiar, M., Nilipour, R., \& Weekes, B. S. (2013). Predictors of timed picture naming in Persian. Behavior Research Methods, 45(3), 834-841.

Berman, S., Friedman, D., Hamberger, M., \& Snodgrass, J. G. (1989). Developmental picture norms: Relationships between name agreement, familiarity, and visual complexity for child and adult ratings of two sets of line drawings. Behavior Research Methods, 21(3), 371-382.

Bonin, P., Méot, A., Lagarrigue, A., \& Roux, S. (2015). Written object naming, spelling to dictation, and immediate copying: different tasks, different pathways? Quarterly Journal of Experimental Psychology, 68, 1268-1294.

Bonin, P., Méot, A., Laroche, B., Bugaiska, A., \& Perret, C. (2019). The impact of image characteristics on written naming in adults. Reading and Writing, 32(1), 13-31.

Bonin, P., Peereman, R., Malardier, N., Méot, A., \& Chalard, M. (2003). A new set of 299 pictures for psycholinguistic studies: French norms for name agreement, image agreement, conceptual familiarity, visual complexity, image variability, age of 
657

658

659

660

661

662

663

664

665

666

667

668

669

670

671

672

673

674

675

676

677

678

679

acquisition, and naming latencies. Behavior Research Methods, Instruments, \& Computers, 35, 158-167.

Bramão, I., Reis, A., Petersson, K. M., \& Faísca, L. (2011). The role of color information on object recognition: A review and meta-analysis. Acta Psicológica, 138(1), 244-253.

Brodeur, M. B., Dionne-Dostie, E., Montreuil, T., \& Lepage, M. (2010). The Bank of Standardized Stimuli (BOSS), a new set of 480 normative photographs of objects to be used as visual stimuli in cognitive research. PloS One, 5(5), e10773.

Brodeur, M. B., Guérard, K., \& Bouras, M. (2014). Bank of standardized stimuli (BOSS) phase II: 930 new normative photographs. PloS One, 9(9), e106953.

Brodie, E. E., Wallace, A. M., \& Sharrat, B. (1991). Effect of surface characteristics and style of production on naming and verification of pictorial stimuli. American Journal of Psychology, 104, 517-545.

Callaghan, T. C. (2000). Factors affecting children's graphic symbol use in the third year: Language, similarity, and iconicity. Cognitive Development, 15(2), 185-214.

Cannard, C., Blaye, A., Scheuner, N., \& Bonthoux, F. (2005). Picture naming in 3-to 8-year-old French children: Methodological considerations for name agreement. Behavior Research Methods, 37(3), 417-425.

Cannard, C., Bonthoux, F., Blaye, A., Scheuner, N., Schreiber, A. C., \& Trinquart, J. (2006). BD2I: Normes sur l'identification de 274 images d'objets et leur mise en relation chez l'enfant français de 3 à 8 ans. L'Année Psychologique, 106(3), 375-396.

Cycowicz Y.M., Friedman, D., Rothstein, M., \& Snodgrass, J.G. (1997). Picture naming by young children: norms for name agreement, familiarity, and visual complexity. Journal of Experimental Child Psychology, 65, 171-237.

Peer) reviewing PDF | (2019:02:35015:1:1:NEW 17 Jul 2019) 
680 D'Amico, S., Devescovi, A., \& Bates, E. (2001). Picture naming and lexical access in Italian 681 children and adults. Journal of Cognition and Development, 2(1), 71-105.

682 Defior, S., Jiménez-Fernández, G., \& Serrano, F. (2009). Complexity and lexicality effects on 683 the acquisition of Spanish spelling. Learning and Instruction, 19(1), 55-65.

684 Delacre, M., Lakens, D., Mora, Y., \& Leys, C. (2018). Taking Parametric Assumptions Seriously 685 Arguments for the Use of Welch's F-test Instead of the Classical F-test in One-way 686 ANOVA. PsyArXiv.

687 Dimitropoulou, M., Duñabeitia, J. A., Blitsas, P., \& Carreiras, M. (2009). A standardized set of 688260 pictures for Modern Greek: Norms for name agreement, age of acquisition, and $689 \quad$ visual complexity. Behavior Research Methods, 41, 584-589.

690 Duchon, A., Perea, M., Sebastián-Gallés, N., Martí, A., \& Carreiras, M. (2013). EsPal: One-stop 691 shopping for Spanish word properties. Behavior Research Methods, 45(4), 1246-1258.

692 Duñabeitia, J. A., Crepaldi, D., Meyer, A. S., New, B., Pliatsikas, C., Smolka, E., \& Brysbaert, 693 M. (2018). MultiPic: A standardized set of 750 drawings with norms for six European

694 languages. The Quarterly Journal of Experimental Psychology, 71(4) 808-816.

695 Forsythe, A., Street, N., \& Helmy, M. (2017). Revisiting Rossion and Pourtois with new ratings 696

697 for automated complexity, familiarity, beauty, and encounter. Behavior Research Methods, 49(4), 1484-1493.

698

Ganea, P. A., Pickard, M. B., \& DeLoache, J. S. (2008). Transfer between picture books and the 699 real world by very young children. Journal of Cognition and Development, 9(1), 46-66.

700 Goikoetxea, E. (2000). Frecuencia de producción de las respuestas a 52 categorías verbales en 701 niños de primaria. Psicológica, 21(1), 61-89. 
702 Harwell, M. R., Rubinstein, E. N., Hayes, W. S., \& Olds, C. C. (1992). Summarizing Monte

703 Carlo results in methodological research: The one- and two-factor fixed effects anova 704 cases. Journal of Educational Statistics, 17 (4), 315-339.

705 Humphreys, G. W., \& Riddoch, M. J. (2006). Features, objects, action: The cognitive neuropsy706 707 chology of visual object processing, 1984-2004. Cognitive Neuropsychology, 23(1), 156-

708

709

710

711

712 713

714 715

716

717

718

719

720

721

722 183.

Kremin, H., Akhutina, T., Basso, A., Davidoff, J., De Wilde, M., Kitzing, P., Lorenz, A., Perrier, D., van der Sandt-Koenderman, M., Vendrell, J, \& Weniger, D. (2003). A cross-linguistic data bank for oral picture naming in Dutch, English, German, French, Italian, Russian, Spanish, and Swedish (PEDOI). Brain \& Cognition, 53, 243-246.

Levelt, W. J. M., Roelofs, A., \& Meyer, A. S. (1999). A theory of lexical access in speech production. Behavioral and Brain Sciences, 22, 1-75.

Lloyd-Jones, T. J., \& Nakabayashi, K. (2009). Independent effects of colour on object identification and memory. The Quarterly Journal of Experimental Psychology, 62(2), 310-322.

Martínez, J. A., \& García, M. E. (2004). Diccionario de frecuencias del castellano escrito en niños de 6 a 12 años [Dictionary of frequencies of written Spanish in 6- to 12-year-old children]. Salamanca: Universidad Pontificia de Salamanca.

Martínez, N., Matute, H., \& Goikoetxea, E. (2019). PicPsy: A new standardized bank of 106 photographs and line-drawings for Spanish-speaking children and adults. Submitted for publication. 
723 Moreno-Martínez, F. J., \& Montoro, P. R. (2012). An ecological alternative to Snodgrass \& 724 Vanderwart: 360 high quality colour images with norms for seven psycholinguistic

725 variables. PloS One, 7(5), e37527.

726 Navarrete, E., Arcara, G., Mondini, S., \& Penolazzi, B. (2019). Italian norms and naming

727 latencies for 357 high quality color images. PloS One, 14: e0209524.

728 Nishimoto, T., Miyawaki, K., Ueda, T., Une, Y., \& Takahashi, M. (2005). Japanese normative 729 set of 359 pictures. Behavior Research Methods, 37, 398-416.

730 Nisi, M., Longoni, A. M., \& Snodgrass, J. G. (2000). Misure italiane per l'accordo sul nome,

731

732

733

734

735

736

737

738

739

740

741

742

743

744 745 familiarità ed età di acquisizione, per le 260 figure di Snodgrass e Vanderwart (1980). Giornale Italiano di Psicologia, 27(1), 205-220.

O’Sullivan, M., Lepage, M., Bouras, M., Montreuil, T., \& Brodeur, M. B. (2012). NorthAmerican norms for name disagreement: Pictorial stimuli naming discrepancies. PloS One, 7(10), e47802.

Perfetti, C. A., Finger, E., \& Hogaboam, T. W. (1978). Sources of vocalization latency differences between skilled and less skilled young readers. Journal of Educational Psychology, 70(5), 730-739.

Perret, C., \& Bonin, P. (2018). Which variables should be controlled for to investigate picture naming in adults? A Bayesian meta-analysis. Behavior Research Methods, 1-13.

Pind, J., Jónsdóttir, H., Tryggvadóttir, H.B., \& Jónsson, F. (2000). Icelandic norms for the Snodgrass and Vanderwart (1980) pictures: Name and image agreement, familiarity, and age of acquisition. Scandinavian Journal of Psychology, 41, 41-48.

Piñeiro, A., Manzano, M., \& Reigosa, V. (1999). Estandarización de un conjunto de 257 figuras en niños de habla hispana cubanos. Cognitiva, 11(2), 215-242.

Peer) reviewing PDF | (2019:02:35015:1:1:NEW 17 Jul 2019) 
746 Pierroutsakos, S. L., \& DeLoache, J. S. (2003). Infants' manual exploration of pictorial objects 747 varying in realism. Infancy, 4, 141-156.

748 Pompéia, S., Miranda, M. C., \& Bueno, O. F. A. (2001). A set of 400 pictures standardized for 749 Portuguese: norms for name agreement, familiarity and visual complexity for children 750 and adults. Arquivos de Neuro-psiquiatria, 59(2B), 330-337.

751 Price, C. J., \& Humphreys, G. W. (1989). The effects of surface detail on object categorization 752 and naming. The Quarterly Journal of Experimental Psychology, 41(4), 797-827.

753

754

755

756

757

758

759

760

761

762

763

764

765

766

767

Raman, I., Raman, E., \& Mertan, B. (2014). A standardized set of 260 pictures for Turkish: norms of name and image agreement, age of acquisition, visual complexity, and conceptual familiarity. Behavior Research Methods, 46, 588-595.

Riddoch, J., \& Humphreys, G. W. (2001) Object recognition. In B. Rapp (Ed.), The handbook of cognitive neuropsychology: What deficits reveal about the human mind (pp. 45-74). New York, NY: Psychology Press.

Roelofs, A., \& Ferreira, V. S. (in press). The architecture of speaking. In P. Hagoort (Ed.), Human language: From genes and brains to behavior. Cambridge, MA: MIT Press.

Rossion, B., \& Pourtois, G. (2004). Revisiting Snodgrass and Vanderwart's object pictorial set: The role of surface detail in basic-level object recognition. Perception, 33(2), 217-236.

Russo, N., Hagmann, C. E., Andrews, R., Black, C., Silberman, M., \& Shea, N. (2018). Validation of the C.A.R.E. stimulus set of 640 animal pictures: Name agreement and quality ratings. PLOS ONE, 13: e0192906.

Salmon, J. P., Matheson, H. E., \& McMullen, P. A. (2014). Photographs of manipulable objects are named more quickly than the same objects depicted as line drawings: Evidence that 
photographs engage embodiment more than line drawings. Frontiers in Psychology, 5, 769 1187.

770 Schmetz, E., Magis, D., Detraux, J. J., Barisnikov, K., \& Rousselle, L. (2019). Basic visual

771

772

773

774

775

776

777

778

779

780

781

782

783

784

785

786

787

788

789 perceptual processes in children with typical development and cerebral palsy: The processing of surface, length, orientation, and position. Child Neuropsychology, 25(2), $232-262$.

Sanfeliu, M. C., \& Fernandez, A. (1996). A set of 254 Snodgrass-Vanderwart pictures standardized for Spanish: Norms for name agreement, image agreement, familiarity, and visual complexity. Behavior Research Methods, Instruments, \& Computers, 28, 537- 555.

Saryazdi, R., Bannon, J., Rodrigues, A., Klammer, C., \& Chambers, C. G. (2018). Picture perfect: A stimulus set of 225 pairs of matched clipart and photographic images normed by Mechanical Turk and laboratory participants. Behavior Research Methods, 1-13.

Shao, Z., \& Stiegert, J. (2016). Predictors of photograph naming: Dutch norms for 327 photographs. Behavior Research Methods, 48(2), 577-584.

Shannon, C. E., \& Weaver, W. (1949). The mathematical theory of communication. In C. E. Shannon \& W. Weaver (Eds.), The Mathematical Theory of Communication. Urbana, IL: University of Illinois Press.

Simcock, G., \& DeLoache, J. S. (2006). The effects of iconicity on re-enactment from picture books by 18- to 30-month-old children. Developmental Psychology, 42(6), 1352-1357.

Sirois, M., Kremin, H., \& Cohen, H. (2006). Picture-naming norms for Canadian French: Name agreement, familiarity, visual complexity, and age of acquisition. Behavior Research Methods, 38(2), 300-306. 
790 Snodgrass, J. G., \& Vanderwart, M. (1980). A standardized set of 260 pictures: norms for name 791 agreement, image agreement, familiarity, and visual complexity. Journal of Experimental 792 Psychology: Human, Learning \& Memory, 6, 174-215.

793 Tiku, M. (1971). Power function of the f-test under non-normal situations. Journal of the 794 American Statistical Association, 66, 913-916.

795 Tsaparina, D., Bonin, P., \& Méot, A. (2011). Russian norms for name agreement, image 796 agreement for the colorized version of the Snodgrass and Vanderwart pictures and age of

797

798 acquisition, conceptual familiarity, and imageability scores for modal object names.

\section{9} 800 Behavior Research Methods, 43, 1085-1099.

Uttl, B., Graf, P., \& Santacruz, P. (2006). Object color effects identification and repetition priming. Scandinavian Journal of Psychology, 47, 313-325. en normen voor plaatjes. Nederlands Tijdschrift voor de Psychologie, 38, 236-241.

Vernon, D., \& Lloyd-Jones, T. (2003). The role of the colour implicit and explicit memory 804 performance. The Quarterly Journal of Experimental Psychology, 56A, 779-802.

Wang, L., Chen, C. W., \& Zhu, L. (2014). Picture norms for Chinese preschool children: Name agreement, familiarity, and visual complexity. PloS One, 9(3), e90450.

808

Weissgerber, T. L., Savic, M., Winham, S. J., Stanisavljevic, D., Garovic, V. D., \& Milic, N. M. (2017). Data visualization, bar naked: A free tool for creating interactive graphics. Journal of Biological Chemistry, 292(50), 20592-20598.

810 Weekes, B. S., Shu, H., Hao, M., Liu, Y., \& Tan, L. H. (2007). Predictors of timed picture naming in Chinese. Behavior Research Methods, 39, 335-342. 
812 Wechsler, D. (2003). Wechsler intelligence scale for children, 4th edition (WISC-IV). San

813 Antonio, TX: Psychological Corporation.

814 
817 measures ANOVA, the Friedman test, and the non-parametric alternative to $t$-test for paired 818 samples, the Wilcoxon's signed rank test, and we found similar results. Significant differences

819 were found between line drawings and photographs in $H$ index, $\chi_{F}^{2}(1)=5.23, p=.022$,

820 percentage of modal name, $\chi_{F}^{2}(1)=5.55, p=.018$, percentage of incorrect alternative names, $W_{s}$ $821=1262, p=.011$, and visual complexity, $W_{s}=2053, p=.028$, but in contrast to the parametric 822 tests, the differences were significant for the TOT responses, with higher percentage of TOT 823 responses, $W_{s}=4.5, p=.019$, in photographs than in line-drawings.

825 Significant differences were found between line drawings and photographs in $H$ index, $\chi_{F}^{2}(1)=$ $8268.67, p=.003$, percentage of modal name, $\chi_{F}^{2}(1)=6.67, p=.010$, percentage of incorrect

827 alternative names, $W_{s}=1166, p<.001$, percentage of TOT responses, $W_{s}=404, p=.002$, and 828 visual complexity, $W_{s}=290.50, p<.001$. 
Figure 1

Examples of picture pairs in line drawing and photograph format

The photographs were retrieved from https://pixabay.com under a CCO license. Attribution is not required. The line drawings were created by one of the authors.

A

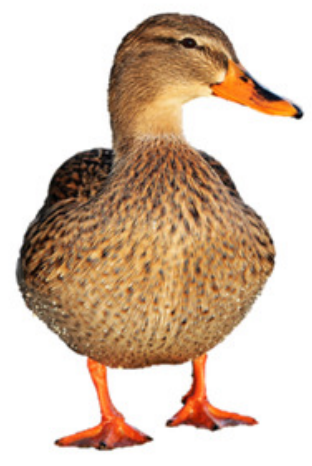

D

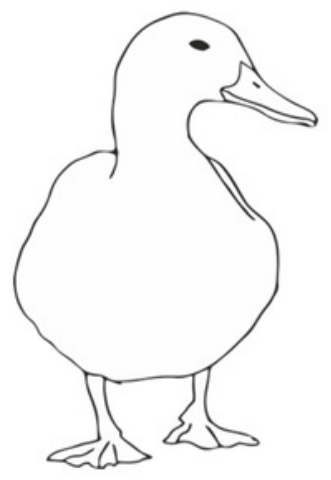

B

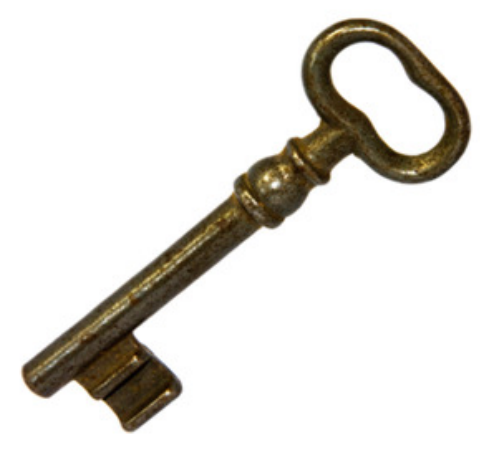

$E$

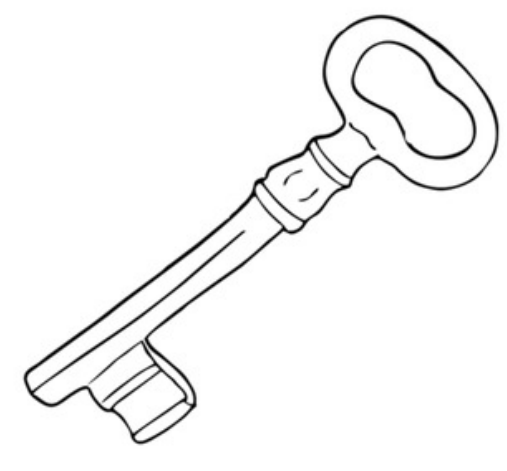

C

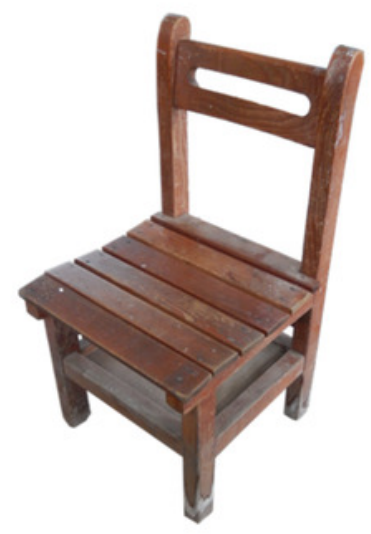

$\mathrm{F}$

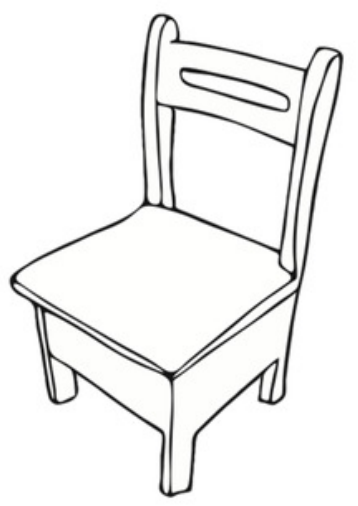


Figure 2

Distribution of picture naming measures in Experiment 1, displayed as violin plots by picture format.

A

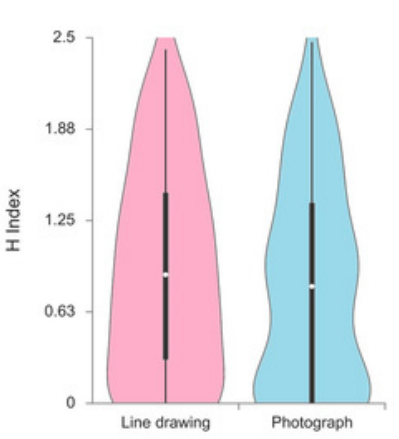

B

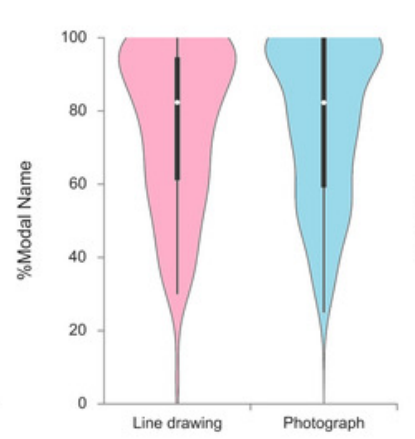

C

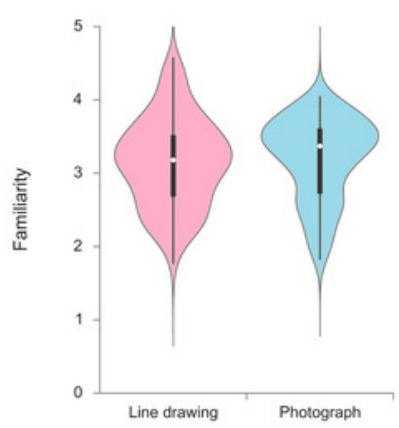

D

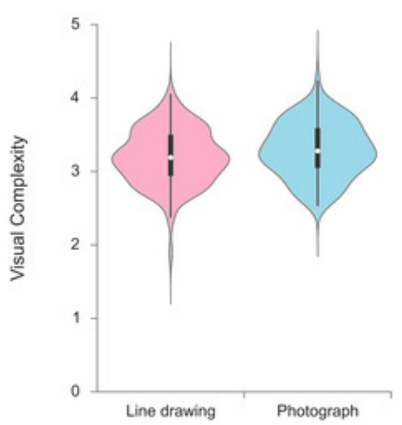


Figure 3

Distribution of picture naming measures in Experiment 2, displayed as violin plots according to picture format

A

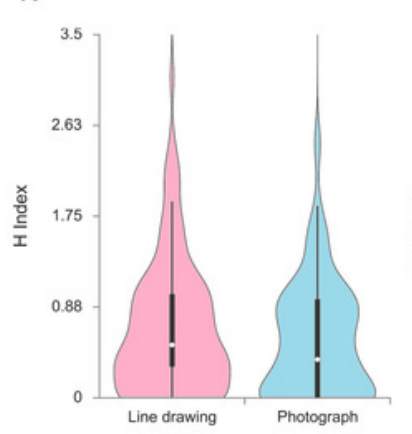

B

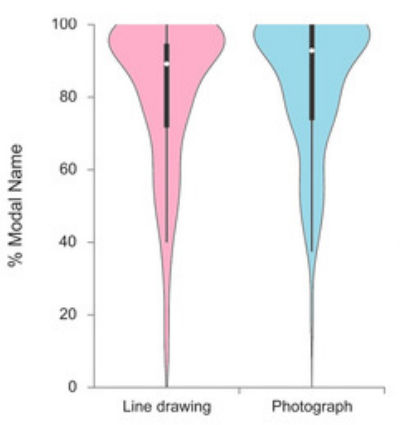

C

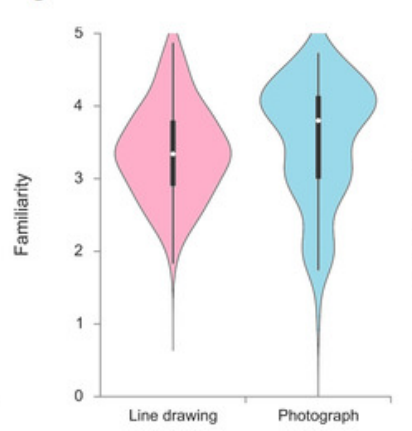

D

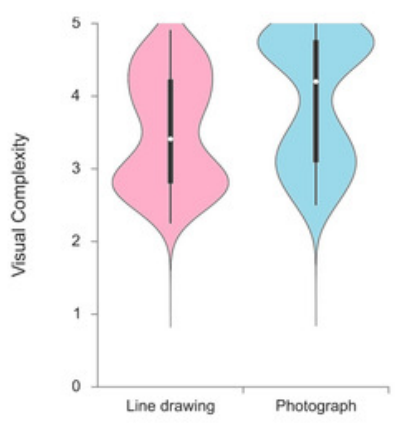




\section{Table $\mathbf{1}$ (on next page)}

Descriptive statistics of the psycholinguistic variables of the stimuli in Experiment 1. 
1 Table 1

2 Descriptive statistics of the psycholinguistic variables of the stimuli in Experiment 1

\begin{tabular}{clcc}
\hline Variable & \multicolumn{1}{c}{ Database } & $M(S D)$ & Range \\
\hline Linguistic variables & & & \\
Lexical frequency & Martínez \& García (2004) & $127.82(237.68)$ & $2.51-1,580.43$ \\
Length & & $4.81(1.66)$ & $3-11$ \\
Fubjective ratings & & & \\
Familiarity & Duchon et al. (2013) & $6.12(0.64)$ & $3.56-7$ \\
Imagination & Duchon et al. (2013) & $6.13(0.51)$ & $4.57-6.85$ \\
Concreteness & Duchon et al. (2013) & $5.83(0.64)$ & $3.74-6.77$ \\
Age of acquisition & Alonso et al. (2015) & $4.23(1.29)$ & $2.32-7.36$ \\
\hline
\end{tabular}




\section{Table 2 (on next page)}

Means (and standard deviations) according to picture format in Experiment 1. 
$1 \quad$ Table 2

2 Means (and standard deviations) according to picture format in Experiment 1

\begin{tabular}{lcc}
\hline \multicolumn{1}{c}{ Variable } & Line drawing & Photograph \\
\hline Name agreement & $0.90(0.71)$ & $0.82(0.71)$ \\
$H$ ind. & $75.47(22.18)$ & $77.33(21.52)$ \\
Modal Name (\%) & & \\
Alternative names & $14.30(17.74)$ & $12.43(15.92)$ \\
Incorrect names (\%) & $0.85(5.62)$ & $0.82(4.56)$ \\
Equivocal names (\%) & $9.38(15.47)$ & $9.42(15.46)$ \\
Correct names (\%) & & \\
Unknown responses & $4.34(8.56)$ & $1.38(10.07)$ \\
Don't Know the Name (\%) & $0.80(3.07)$ & $1.11(5.05)$ \\
Don't Know the Object (\%) & $0.25(1.12)$ & $3.19(0.56)$ \\
Tip-Of-the-Tongue (\%) & & $3.29(0.37)$ \\
Subjective scales & $3.15(0.61)$ & \\
Familiarity & $3.20(0.37)$ & \\
Visual Complexity & & \\
\end{tabular}

3 
Table 3 (on next page)

Descriptive statistics of the psycholinguistic variables in Experiment 2. 
1 Table 3

2 Descriptive statistics of the psycholinguistic variables in Experiment 2

\begin{tabular}{clcc}
\hline Variable & \multicolumn{1}{c}{ Database } & $M(S D)$ & Range \\
\hline Linguistic variables & & & \\
Lexical frequency & Martínez \& García (2004) & $148.52(181.68)$ & $2.09-808.62$ \\
Length & & $5.04(1.70)$ & $2-10$ \\
Subjective ratings & & & \\
Familiarity & Duchon et al. (2013) & $5.91(0.72)$ & $3.57-6.91$ \\
Imagination & Duchon et al. (2013) & $6.04(0.58)$ & $4.86-6.75$ \\
Concreteness & Duchon et al. (2013) & $5.87(0.63)$ & $4.72-6.97$ \\
Age of acquisition & Alonso et al. (2015) & $4.31(1.36)$ & $1.96-7.56$ \\
\hline
\end{tabular}




\section{Table 4 (on next page)}

Means (and standard deviations) according to picture format in Experiment 2. 
$1 \quad$ Table 4

2 Means (and standard deviations) according to picture format in Experiment 2

\begin{tabular}{lcc}
\hline \multicolumn{1}{c}{ Variable } & Line drawing & Photograph \\
\hline Name agreement & & \\
$H$ ind. & $0.68(0.64)$ & $0.55(0.56)$ \\
Modal Name (\%) & $81.07(21.02)$ & $83.96(18.53)$ \\
Alternative names & $11.94(16.49)$ & $9.21(14.42)$ \\
Incorrect names (\%) & $1.02(9.01)$ & $0.19(1.44)$ \\
Equivocal names (\%) & $5.97(11.00)$ & $6.64(13.65)$ \\
Correct names $(\%)$ & & $1.76(4.03)$ \\
Unknown responses & $1.80(4.55)$ & $1.94(4.67)$ \\
Don't Know the Name (\%) & $1.54(4.93)$ & $1.53(3.33)$ \\
Don't Know the Object $(\%)$ & $2.61(4.20)$ & \\
Tip-Of-the-Tongue $(\%)$ & & $3.53(0.83)$ \\
Subjective scales & $3.37(0.65)$ & $3.93(0.86)$ \\
Familiarity & $3.51(0.77)$ & \\
Visual Complexity & & \\
\hline
\end{tabular}

3 